\title{
Study on Metallo-Beta Lactamase Producing Pseudomonas Species in Clinical Isolates of a Tertiary Care Hospital of Western Odisha
}

\author{
Shuvankar Mukherjee ${ }^{1}$, Suchitra Mishra², Shreekant Tiwari ${ }^{3}$ \\ ${ }^{1}$ Department of Microbiology, Hitech Medical College, Rourkela, Odisha, India. ${ }^{2}$ Department of Microbiology, Hitech \\ Medical College, Rourkela, Odisha, India. ${ }^{3}$ Department of Microbiology, Hitech Medical College, Rourkela, Odisha, India.
}

\section{ABSTRACT}

\section{BACKGROUND}

Pseudomonas species are responsible for $10 \%$ of hospital acquired infection especially in an ICU set up and in burn patients. Metallo-beta lactamase production is the most common mechanism of resistance to carbapenem which is the most commonly used drug to treat Pseudomonas. Local prevalence of MBL producing Pseudomonas is important information to both microbiologist and clinician to formulate hospital infection control strategy. This cross-sectional descriptive study was conducted in a tertiary care hospital of western Odisha to detect MBL prevalence among clinical isolates of Pseudomonas species.

\section{METHODS}

187 Pseudomonas strains (165 P. aeruginosa and 22 P. putida) isolated in different clinical samples in Vitek 2 system were checked for imipenem resistance (MIC $>8$ $\mu \mathrm{l} / \mathrm{ml}$ ). All imipenem resistance strains were checked for MBL production by combined disc test with imipenem, and MBL production was confirmed by MBL E test.

\section{RESULTS}

Among 187 Pseudomonas strains 12.20\% were carbapenem resistant and 9\% were MBL producing. About $74 \%$ of carbapenem resistant Pseudomonas strains were MBL positive. MBL positivity rate was much higher in Pseudomonas putida $(27.20 \%)$ compared to Pseudomonas aeruginosa (7\%) and in ICU (14.20\%) compared to IPD $(9.20 \%)$ or OPD $(6.80 \%)$. Colistin was the most effective $(97 \%)$ antibiotic against MBL producing Pseudomonas.

\section{CONCLUSIONS}

It is better to prevent MBL Pseudomonas than to cure it as most of the antibiotics were found to be ineffective against it. In our study MBL production rate in clinical isolate of Pseudomonas was low (9\%) compared to other studies in India.

\section{KEY WORDS}

Pseudomonas, Carbapenem, MBL, Positivity.
Corresponding Author:

Suchitra Mishra,

Department of Microbiology,

Hitech Medical College,

Rourkela, Odisha, India.

E-mail:shmu963@gmail.com

DOI: $10.14260 / \mathrm{jemds} / 2020 / 335$

Financial or Other Competing Interests: None.

How to Cite This Article:

Mukherjee S, Mishra S, Tiwari S. Study on metallo-beta lactamase producing Pseudomonas species in clinical isolates of a tertiary care hospital of Western Odisha. J. Evolution Med. Dent. Sci. 2020;9(19): 1533-1538, DOI:

10.14260/jemds/2020/335

Submission 11-03-2020,

Peer Review 24-04-2020,

Acceptance 30-04-2020,

Published 11-05-2020.

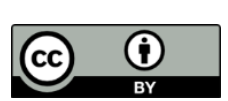




\section{BACKGROUND}

"In Wine there is Truth, in Beer there is Strength, in Water there are Pseudomonas." (adaption of German proverb) Pseudomonas are the gram-negative bacilli that are strict aerobes, motile with one or two flagella, utilise glucose oxidatively, and are oxidase positive. It belongs to Pseudomonadaceae family and in molecular taxonomy to fluorescent group of $r$ RNA group I. ${ }^{1}$ Another members of this Fluorescent group are Pseudomonas fluorescence and Pseudomonas putida that are rarely involved in clinical diseases in human. ${ }^{2}$ With the help of different virulent factors like pyocyanin, exotoxin A, exoenzyme $S$, protease, phospholipase, rhamnolipids they produce both community acquired infections like otitis externa, keratitis, varicose vein ulcer and hospital acquired infection like Catheter associated urinary tract infection (CAUTI), Ventilator associated pneumonia (VAP), burn infection, bedsore, septicaemia and necrotising pneumonia in cystic fibrosis patients etc. ${ }^{2}$ They have highly evolved Quorum sensing mechanism by which they can easily form biofilm and prevent attack of antibiotic ${ }^{2}$. Most important factor that makes it so much dominant in hospital environment that it can resist or even can utilise some disinfectants/ antiseptics like cetrimide for their nutrition, ${ }^{1}$ so they easily grow in hospital environment and ICU. They are responsible for $10 \%$ of all hospital acquired infection. ${ }^{2}$ Aminoglycoside (gentamicin, amikacin, tobramycin), antipseudomonal penicillin (piperacillin, ticarcillin) and cephalosporin like Ceftazidime are used to treat Pseudomonal infection but resistance against these antibiotics are common today. ${ }^{2}$

Beta lactamase destroy beta lactam ring of antibiotic and make them ineffective against Pseudomonas. ${ }^{3}$ carbapenem is the drug of choice in extended spectrum beta lactamase producing Pseudomonas. ${ }^{3}$ This was derived from thienamycin, a naturally derived product of Streptomyces cattleya. 4 Ertopenem, Doripenem, imipenem, Meropenem and Faropenem are example of carbapenem but imipenem and Meropenem are most commonly used carbapenem in India. ${ }^{4}$ With the progress of time irrational and inappropriate use of carbapenem led to emergence of carbapenem resistant Pseudomonas- first in Japan in $1991^{5}$ and then in different part of the world. In India first case of MBL producing Pseudomonas was reported in 2002.6,7 Mechanism of carbapenem resistance are mainly three types, first due to increase expression of porin in cell wall, second due to increase activity of efflux pump and third-production of metallo-beta lactamase. ${ }^{7}$

Metallo-beta lactamase production is the most common mechanism of carbapenem resistance ${ }^{7}$. Metallo-beta lactamase is a zinc dependent enzyme belonging to Ambler class $\mathrm{B}$ that can hydrolyse all beta lactam antibiotics including carbapenem ${ }^{8}$. Ambler class A,C,D. beta lactamases use serine as active site so they can be easily degraded by beta lactamase inhibitor like clavulanic acid or sulbactam ${ }^{8}$. But metallo-beta lactamase cannot be inhibited by clavulanic acid or sulbactum so MBL producing Pseudomonas is now emerging as a nightmare for treating physician. Besides that, resistance determinant of MBL is located in highly mobile genetic element allowing easy dissemination from patient to patient or even from patient to health care providers. ${ }^{2}$ So prevention is the always better option than treatment of MBL Pseudomonas infection. Clinician in every hospital should know the local prevalence of MBL producing Pseudomonas to formulate proper antibiotic policy and hospital infection control strategy to prevent outbreak of this dangerous superbug. Keeping it in mind we have conducted a research to find out MBL positivity rate in clinical isolates of Pseudomonas in a tertiary care hospital of Western Odisha.

\section{METHODS}

This is a descriptive cross-sectional study conducted in the Department of Microbiology, of Hitech Medical College, Rourkela, Odisha, over a period of 1 year (March 2019 to Feb. 2020). Depending upon site of infection samples were collected like urine, pus, sputum, BAL, ear swab etc in sterile container.

\section{Sample Processing}

All samples were inoculated immediately into Blood agar and MacConkey agar media (HiMedia, Mumbai) and incubated for 18-24 hrs. At $37^{\circ} \mathrm{C}$ in incubator. Next day growth was observed, and gram stain was performed. All the positive growth which was oxidase positive selected and put in Vitek 2 identification system (Biomerieux). Identification and antibiogram of oxidase positive growth was done in fully automated Vitek 2. Carbapenem resistance was suspected when either imipenem or meropenem was resistant (mic $>8$ $\mu \mathrm{l} / \mathrm{ml}$ ).MBL production was tested in all carbapenem resistant Pseudomonas species by Combined disc test with imipenem and was confirmed by MBL- E test.

\section{Combined Disc Test ${ }^{5}$}

Two $10 \mu \mathrm{g}$ IPM disks were put on the MHA plate seeded with the test organism. $10 \mu \mathrm{L}$ of EDTA solution $(750 \mu \mathrm{g})$ was added to one of them. The plate was incubated for 16-18 hrs at $35^{\circ} \mathrm{C}$. If the increase in inhibition zone with the IPM + EDTA disk was $>7 \mathrm{~mm}$ than the IPM disk alone, it was suspected as MBL positive.

\section{MBL Epsilometer Test (E-Test) ${ }^{5}$}

The MBL E-strip with seven-dilution range of IPM (4-256 $\mu \mathrm{g} / \mathrm{mL}$ ) in one side and IPM plus EDTA (1-64 $\mu \mathrm{g} / \mathrm{mL})$ on another side was put on MHA plate seeded with test organism. The plate was then incubated in incubator at $35^{\circ} \mathrm{C}$ for 18-20 hrs. MIC ratio of IPM/IPM + EDTA of $>8$, or reduction of IPM MIC by $>3 \log 2$ dilutions in the presence of EDTA confirmed MBL production. 


\section{RESULTS}

Total 187 isolates of Pseudomonas species were isolated. A mong which 149 were from urine and 20 were from pus. 13 were from another sites like sputum, ear swab, bronchoalveolar lavage (BAL), endotracheal tube (ET) aspirate etc.
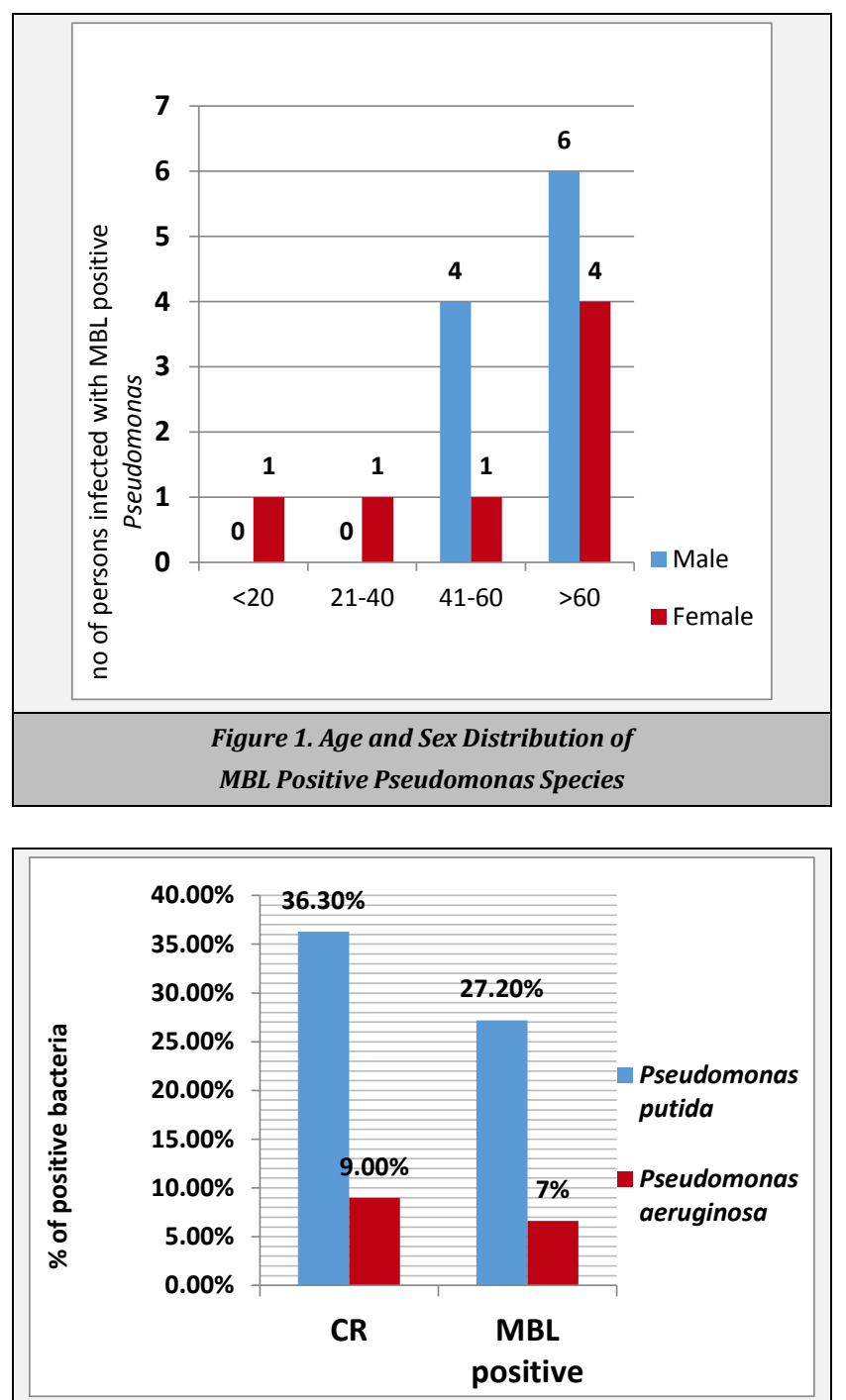

Figure 2. Carbapenem and MBL Positivity Rate in Different Species of Pseudomonas

Among 187 samples 165 were $P$. aeruginosa and 87 were $P$. putida. 87 Pseudomonas strains were isolated from OPD, 65 were from IPD and 35 were from ICU. Carbapenem resistance rate was $12.2 \%$ and MBL positivity rate was $9 \% .74 \%$ of carbapenem resistance Pseudomonas was MBL positive. Among 17 MBL producing strains 10 were from male patients and 7 were from female patients. Age and sex distribution of MBL positive patients was showed in Fig 1. Carbapenem resistance and MBL positivity rate of two species of Pseudomonas was showed in Fig 2. Sample wise CR and MBL positivity rate was depicted in Fig 3. Ward wise CR and MBL positivity rate in Pseudomonas was showed in Fig 4. Antibiogram of MBL positive and MBL negative Pseudomonas species was compared in Table 1.
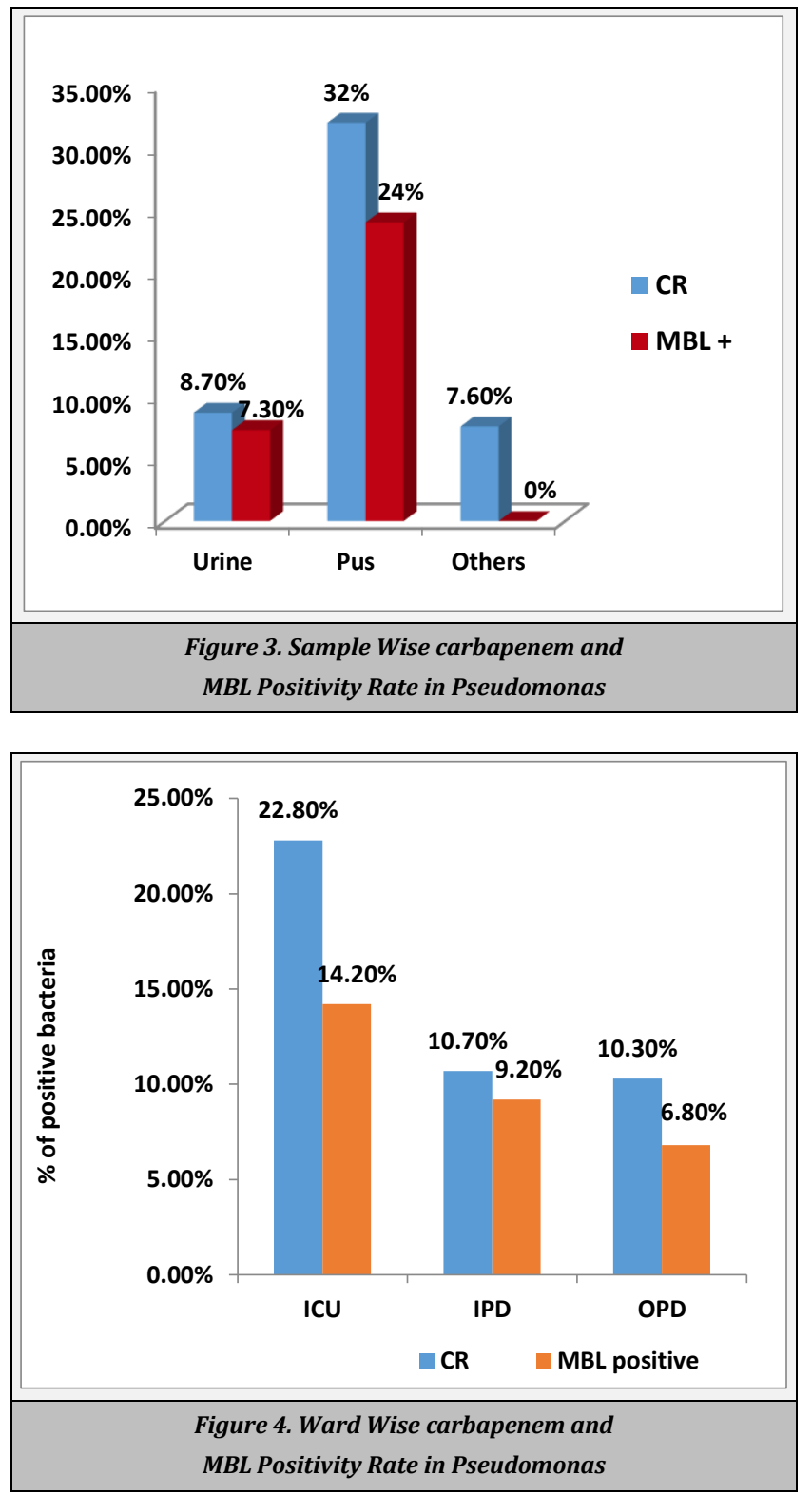

\begin{tabular}{|ccc|}
\hline Antibiotics & $\begin{array}{c}\text { Sensitivity Shown } \\
\text { by MBL Positive } \\
\text { Pseudomonas spp. }\end{array}$ & $\begin{array}{c}\text { Sensitivity Shown by } \\
\text { MBL Negative } \\
\text { Pseudomonas spp. }\end{array}$ \\
Ampicillin & 10.4 & (\%) \\
Amoxycillin-clavulanic acid & 18.10 & 40.29 \\
Piperacillin-Tazobactam & 42.12 & 66.76 \\
Cefuroxime & 2.3 & 90 \\
Ceftazidime & 20 & 40.78 \\
Cefoperazone sulbactam & 40 & 66.7 \\
Cefepime & 2.21 & 88.82 \\
Amikacin & 25.10 & 67.29 \\
Gentamicin & 21.21 & 93.64 \\
Ciprofloxacin & 26.84 & 83.64 \\
Tigecycline & 12.52 & 66.29 \\
Nitrofurantoin (urine) & 15.57 & 80.64 \\
Colistin & 97 & 75.29 \\
Trimethoprim/Sulfamethoxazole & 22.67 & 98.11 \\
\hline Table 1. Antibiotic Sensitivity Pattern of Pseudomonas Species \\
\hline \multicolumn{2}{|c}{} \\
\hline
\end{tabular}

\section{DISCUSSION}

Pseudomonas has the ability to grow and multiply in moist environment and equipment including sinks, drain flower vas, hydrotherapy pools, ponds, river and even in distilled water ${ }^{2}$. In hospital setting it can grow in many disinfectants and pharmaceutical product posing serious problem in 
infection control. ${ }^{2}$ Blue green colour pyocyanin produced by $P$. aeruginosa and pyoverdine produced by another Pseudomonas act as virulent factor. With the help of metallobeta lactamase enzyme they become antibiotic resistant ${ }^{9}$. Lots of phenotypic tests are available for detection of metallobeta lactamase production in Pseudomonas. Principal of all those test is the ability of metal ion chelator like EDTA or thiol compound to inhibit activity of MBL. ${ }^{10}$ These tests include Combined disc test (CDT) using EDTA with imipenem or ceftazidime, Double disc synergy test (DDST), EDTA disc potentiation test (PT) using ceftazidime or cefotaxime, Modified Hodge test, Carba NP test, Modified carbapenem inactivation method (mCIM) etc. 5 These all tests are used for screening purpose. Among all these tests CDT showed good sensitivity (79\%) than other tests like DDST (70.8\%) and Disc potentiation test (54.2\%) in a study done by Ranjan et al. ${ }^{5}$

Another studies like Samuelson et al,11 Qu et al,12 Biradar et $\mathrm{al}^{13}$ also had reported that CDT was better than all other tests. It was easy to perform and cheaper and having objective interpretation. ${ }^{5}$ Rit et al ${ }^{14}$ in Kolkata had reported that CDT was having same accuracy with MIC detection. So we had selected CDT as screening test for MBL detection in our study. CDT could be performed using ceftazidime or imipenem but as Pseudomonas might have other resistance mechanism other than MBL production to ceftazidime ${ }^{15}$ we had used imipenem for CDT. For confirmation, PCR analysis of MBL gene was the gold standard but it was not feasible in routine microbiology laboratory in a developing country like India. ${ }^{5}$ In contrast, MBL E test showed good specificity (98\%) in study by Khosravi et $\mathrm{al}^{16}{ }^{16}$ Walsh et $\mathrm{al}^{17}$ and Segal et al ${ }^{18}$. So, we had selected MBL E test as the confirmatory test for MBL detection in our study.

In our study among 187 isolated Pseudomonas strains, $12,20 \%$ were carbapenem resistant which was almost similar with study done by Kanungo et $\mathrm{al}^{19}(10.9 \%)$ but was lower than study done by Rajput et $\mathrm{al}^{3}(17.2 \%)$, Ranjan et $\mathrm{al}^{5}$ $(21.3 \%)$, Choudhary et $\mathrm{al}^{9}(33.88 \%)$, Mishra et al ${ }^{10}(58 \%)$, Biradar et al ${ }^{13}(32 \%)$ and Varaiya et al20 (26\%). Implementation of strict antibiotic policy in our hospital might be responsible for such low level of carbapenem resistance in our study. Among carbapenem resistant Pseudomonas, $73.9 \%$ were MBL producing in our study which was almost same as that of study done by Biradar et $\mathrm{al}^{13}$ (74\%) but was lower than Mishra et al ${ }^{10}(100 \%)$,Chand et al ${ }^{21}$ (94.52\%), Attal et $\mathrm{al}^{22}(88.89 \%)$, Fam et $\mathrm{al}^{23}(87.5 \%)$ and Irfan et $\mathrm{al}^{24}(100 \%)$.In our study MBL positivity rate was $9 \%$ which was lower than Rajput et $\mathrm{al}^{3}(12 \%)$, Kaur et $\mathrm{al}^{8}$ (21.8\%), Choudhary et $\mathrm{al}^{9}(20 \%)$, Mishra et $\mathrm{al}^{10}(58 \%)$, Qu et al $^{12}(9.1 \%)$, Biradar study ${ }^{13}(25 \%)$, Rit et al ${ }^{14}$ (41\%), Behera et $\mathrm{al}^{15}(39.56 \%)$, Khosravi et $\mathrm{al}^{16}(19.5 \%)$, Varaiya et $\mathrm{al}^{20}$ (14.3\%), Navneet et $\mathrm{al}^{25}$ (12\%), Hemlata et $\mathrm{al}^{26}$ (14\%), Castanheira et al ${ }^{27}$ (34\%), Owlia et al ${ }^{28}$ (19.7\%), Manoharan et $\mathrm{al}^{29}(42.6 \%)$, Kumar et $\mathrm{al}^{30}(26.9 \%)$ and Kali et $\mathrm{al}^{31}(22.4 \%)$. Lower MBL positivity rate than our study (9\%) were reported by few studies like Mandiratta et al ${ }^{32}$ (8.2\%), Agarwal et $\mathrm{al}^{33}(8.05 \%)$ Pitout et $\mathrm{al}^{34}$ (7.65\%), Ibukin et al ${ }^{35}$ $(4.12 \%)$ and Choudhary et $\mathrm{al}^{36}(6.12 \%)$. Variation in MBL positivity rate in different studies might be due to several factors like Geographical area, Infection control attitude of the hospital, sample size and and method of testing ${ }^{5}$. But overall in our hospital MBL positivity rate was lower than most of the studies in India and it should be maintained in future by strict hospital infection control and antibiotic policy.

Slight male preponderance was seen in in MBL positive Pseudomonal infection, but it was not significant. Similar Male preponderance was reported by Ranjan et al, 5 Choudhary et al. ${ }^{9}$ and Biradar et $\mathrm{al}^{13}$ but in a study in Nepal ${ }^{37}$ slight female preponderance was noted. Highest no of MBL positive isolates (10 out of 17) came from older age group $(>60)$ in our study. In contrast, Choudhary et al $^{9}$ and Biradar et $\mathrm{al}^{13}$ had reported middle age group (31-60) was the most commonly affected age group. Long hospital stay, frequent hospital admission due to age related problems and relative immunocompromised status of older age group ${ }^{5}$ might be responsible for higher MBL positivity rate in older age group in our study.

MBL positivity in Pseudomonas was highest in pus isolates (24\%) followed by urine isolates (7.3\%).Rajput et $\mathrm{al}^{3}$, Choudhary et al, ${ }^{9}$ Mishra et al al, ${ }^{10}$ Biradar et al $^{13}$ and Chand et $\mathrm{al}^{21}$ had reported similar finding. Wound easily comes in contact of hospital environment leading to easy colonisation compared to bladder that requires catheter manipulation to get infected by Pseudomonas. MBL positivity of Pseudomonas in our study was highest in ICU $(14.20 \%)$ compared to IPD (9.20\%) and OPD (6.80\%).It was consistent with the study by Kaur et $\mathrm{al}^{8}$ but not with the study done by Easwaran et $\mathrm{al}^{4}$ where MBL positivity was highest in IPD followed by ICU. More number of invasive interventions prolong stay in ICU, serious nature of the disease in ICU patients ${ }^{8}$ all might be responsible for high MBL positivity rate in ICU compared to IPD and OPD in our study. Most of the study done in India about MBL production was in Pseudomonas aeruginosa which was the most common species of Pseudomonas. As per our literature search no data was available about MBL positivity in Pseudomonas putida in India. We had found that $27.20 \%$ of P. putida and $7 \%$ of $P$. aeruginosa strains were MBL positive. MBL positivity was about 4 times higher in $P$. putida than $P$. aeruginosa in our study. It was in consistent with a spanish study ${ }^{38}$ where $14 \%$ of $P$. putida and $0.3 \%$ of $P$. aeruginosa was MBL positive. $\mathrm{P}$ putida acts as environmental reservoir of MBL resistance gene and acts as a donor of this gene to $P$. aeruginosa. ${ }^{38}$

In antibiotic sensitivity test, MBL positive Pseudomonas species showed poor sensitivity against most of the antibiotics like ampicillin (10.4\%), amoxiclav (18.10\%), Cefuroxime (2.3\%), Ceftazidime (20\%), Cefoperazone sulbactum (40\%), amikacin $(25.10 \%)$ gentamicin $(21.21 \%)$ ciprofloxacin (26.84\%), cotrimoxazole (22.67\%), Nitrofurantoin $(15.57 \%)$ etc compared to MBL negative strains. This finding was consistent with another studies like Choudhary et al, ${ }^{9}$ Mishra et al ${ }^{10}$ and Biradar et al. ${ }^{13}$ However, in our study sensitivity towards piperacillin tazobactam in MBL positive strains was $42.12 \%$ that was almost same with the study done by Mishra et $\mathrm{al}^{10}(48.42 \%)$, Biradar et $\mathrm{al}^{13}$ (38\%) and Chand et $\mathrm{al}^{21}(47.80 \%)$ and was higher than Choudhary et $\mathrm{al}^{9}(19.5 \%)$. In our study colistin resistance was seen in $3 \%$ of MBL positive strains like study by Choudhary et $\mathrm{al}^{9}(2.7 \%)$ but not like Mishra etal ${ }^{10}$ where very high colistin resistance $(58.95 \%)$ was reported in capital city of Odisha. In contrast, Biradar et $\mathrm{al}^{13}$ from Kashmir had reported that $100 \%$ sensitivity to colistin in MBL positive 
Pseudomonas species. Although colistin is the last resort against MBL positive Pseudomonas it cannot be used randomly due nephrotoxic side effect. ${ }^{13}$

In every hospital MBL positivity in Pseudomonas should be checked by Microbiologist as routine laboratory practice and local prevalence of that superbug should be kept in mind during hospital infection control policy making to prevent outbreak of this highly communicable resistance determinant.

\section{CONCLUSIONS}

MBL producing Pseudomonas is difficult to treat but easy to prevent by proper hospital infection control measures and antibiotic policy. In our study MBL positivity rate $(9 \%)$ in Pseudomonas was lower when compared to most of the similar studies in India. MBL prevalence in Pseudomonas putida $(27.2 \%)$ was four times higher than Pseudomonas aeruginosa (7\%). Colistin was the only antibiotic with good sensitivity (97\%) against this dangerous superbug.

\section{REFERENCES}

[1] Koneman EW, Allen SD, Janda WM, et al. Color Atlas and Textbook of Diagonostic Microbiology. $6^{\text {th }}$ edn. Philadelphia, USA: Lippincott Raven Publishers 1997.

[2] Greenwood D. Medical microbiology. 18 th $^{\text {th }}$ edn. Edinburgh: Churchill Livingstone/Elsevier 2012.

[3] Rajput A, Prajapati B, Chauhan B, et al. Prevalence of Metallo-Betalactamases (MBL) producing Pseudomonas aeruginosa in a tertiary care hospital. Ind J Basic \& App Med Res 2012;1(4):304-8.

[4] Easwaran S, Ramasamy R. Prevalence of metallo $\beta$ lactamases producing Pseudomonas spp. and acinetobacter spp. in a tertiary care teaching hospital. J Drug Discovery Ther 2017;5(7):35-9.

[5] Ranjan S, Banashankari GS, Babu PR. Evaluation of phenotypic tests and screening markers for detection of metallo- $\beta$-lactamases in clinical isolates of Pseudomonas aeruginosa: a prospective study. Medical Journal of Dr. DY Patil University 2015;8(5):599-605.

[6] Peleg AY, Franklin C, Bell JM, et al. Dissemination of the metallo- $\beta$-lactamase gene blaIMP-4 among gramnegative pathogens in a clinical setting in Australia. Clinical Infectious Diseases 2005;41(11):1549-56.

[7] Nandi A, Bhattacharya S, Biswas S, et al. A study on Metallo- $\beta$ lactamase producing imipenem nonsusceptible multi-drug resistant Pseudomonas aeruginosa in different clinical specimens in a tertiary care hospital in Kolkata. J Dent Med Sci 2014;13(6):13-7.

[8] Kaur A, Singh S. Prevalence of Extended Spectrum Betalactamase (ESBL) and Metallobetalactamase (MBL) producing Pseudomonas aeruginosa and acinetobacter baumannii isolated from various clinical samples. Journal of Pathogens 2018;2018:6845985.

[9] Choudhary V, Pal N, Hooja S. Prevalence and antibiotic resistance pattern of Metallo- $\beta$-lactamase-producing Pseudomonas aeruginosa isolates from clinical specimens in a tertiary care hospital. Journal of Mahatma Gandhi Institute of Medical Sciences 2019;24(1):19-22.

[10] Mishra SN, Biswal SR, Behera BK, et al. Detection of prevalence of metallo-beta lactamases in clinical isolates of imipenem resistant Pseudomonas aeruginosa from neonatal septicemia cases in a tertiary hospital in Odisha, India. Int J Contemp Pediatr 2018;5(1):61-6.

[11] Samuelsen O, Buaro L, Giske CG, et al. Evaluation of phenotypic tests for the detection of metallo- $\beta$ lactamase-producing Pseudomonas aeruginosa in a low prevalence country. Journal of Antimicrobial Chemotherapy 2008;61(4):827-30.

[12] Qu TT, Zhang JL, Wang J, et al. Evaluation of phenotypic tests for detection of Metallo- $\beta$-lactamase-producing Pseudomonas aeruginosa strains in China. Journal of Clinical Microbiology 2009;47(4):1136-42.

[13] Biradar S, Roopa C. Prevalence of Metallo-beta lactamase producing Pseudomonas aeruginosa and its antibiogram in a tertiary care centre. Int J Curr Microbiol Appl Sci 2015;4(9):952-6.

[14] Rit K, Chakraborty B, Dey R, et al. Prevalence of Pseudomonas aeruginosa and acinetobacter spp. producing metallo- $\beta$-lactamase in a tertiary care hospital. Journal of Dr. NTR University of Health Sciences 2013;2(1):18-21.

[15] Behera B, Mathur P, Das A, et al. An evaluation of four different phenotypic techniques for detection of metallo$\beta$-lactamase producing Pseudomonas aeruginosa. Indian Journal of Medical Microbiology 2008;26(3):233-7.

[16] Khosravi Y, Loke MF, Chua EG, et al. Phenotypic detection of metallo- $\beta$-lactamase in imipenem-resistant Pseudomonas aeruginosa. The Scientific World Journal 2012;2012:654939.

[17] Walsh TR, Bolmström A, Qwärnström A, et al. Evaluation of a new Etest for detecting metallo- $\beta$-lactamases in routine clinical testing. Journal of Clinical Microbiology 2002;40(8):2755-9.

[18] Segal H, Elisha BG. Use of Etest MBL strips for the detection of carbapenemases in Acinetobacter baumannii. Journal of Antimicrobial Chemotherapy 2005;56(3):598.

[19] Shashikala, Kanungo R, Srinivasan S, et al. Emerging resistance to carbapenems in hospital acquired Pseudomonas infection: a cause for concern. Indian Journal of Pharmacology 2006;38(4):287-8.

[20] Varaiya A, Kulkarni M, Bhalekar P, et al. Incidence of metallo-beta-lactamase-producing Pseudomonas aeruginosa in diabetes and cancer patients. Indian Journal of Pathology and Microbiology 2008;51(2):2003.

[21] Chand AE, Chauhan PS, Sharma S, et al. Prevalence of Metallo-beta-lactamase production in imipenemresistant Pseudomonas in tertiary care center at Kota region. Int J Sci Stud 2016;4(3):87-91.

[22] Attal RO, Basak S, Mallick SK, et al. Metallo-betalactamase producing Pseudomonas aeruginosa: an emerging threat to clinicians. J Clin Diagn Res 2010;4:2691-6.

[23] Fam N, Diab M, Helmi H, et al. Phenotypic detection of metallo- $\beta$-Lactamases and extended spectrum $\beta$ - 
Lactamases among Gram-negative bacterial clinical isolates. Egyptian Journal of Medical Microbiology 2006;15(4):719-30.

[24] Irfan S, Zafar A, Guhar D, et al. Metallo- $\beta$-lactamaseproducing clinical isolates of acinetobacter species and Pseudomonas aeruginosa from intensive care unit patients of a tertiary care hospital. Indian Journal of Medical Microbiology 2008;26(3):243-5.

[25] Navaneeth BV, Sridaran D, Sahay D, et al. A preliminary study on metallo-[beta]-lactamase producing Pseudomonas aeruginosa in hospitalized patients. Indian Journal of Medical Research 2002;116:264-7.

[26] Hemalatha V, Sekar U, Kamat V. Detection of metallo- $\beta$ lactamase producing Pseudomonas aeruginosa in hospitalized patients. Indian J Med Res 2005;122(2):148-52.

[27] Castanheira M, Bell JM, Turnidge JD, et al. Carbapenem resistance among Pseudomonas aeruginosa strains from India: evidence for nationwide endemicity of multiple metallo- $\beta$-lactamase clones (VIM-2,-5,-6, and-11 and the newly characterized VIM-18). Antimicrobial Agents And Chemotherapy 2009;53(3):1225-7.

[28] Owlia P, Saderi H, Karimi Z, et al. Phenotypic detection of Metallo-beta-Lactamase producing Pseudomonas aeruginosa strains isolated from burned patients. Iranian Journal of Pathology 2008;3(1):20-5.

[29] Manoharan A, Chatterjee S, Mathai D, et al. Detection and characterization of metallo-beta lactamases producing Pseudomonas aeruginosa. Indian Journal of Medical Microbiology 2010;28(3):241-4.

[30] Kumar SH, De Anuradha S, Baveja SM, et al. Prevalence and risk factors of metallo $\beta$-lactamase producing Pseudomonas aeruginosa and acinetobacter species in burns and surgical wards in a tertiary care hospital. Journal of Laboratory Physicians 2012;4(1):39-42.

[31] Kali A, Srirangaraj SK, Kumar S, et al. Detection of metallo-beta-lactamase producing Pseudomonas aeruginosa in intensive care units. The Australasian Medical Journal 2013;6(12):686-93.

[32] Mendiratta DK, Deotale V, Narang P. Metallo-betalactamase producing Pseudomonas aeruginosa in a hospital from a rural area. Indian Journal of Medical Research 2005;121(5):701-3.

[33] Agrawal G, Lodhi RB, Kamalakar UP, et al. Study of metallo-beta-lactamases production in clinical isolates of Pseudomonas aerugonisa. Indian J Med Microbiol 2008;26(4):349-51.

[34] Pitout JDD, Gregson DB, Poirel L, et al. Detection of Pseudomonas aeruginosa producing metallo- $\beta$ lactamases in a large centralized laboratory. Journal of Clinical Microbiology 2005;43(7):3129-35.

[35] Ibukun A, Tochukwu N, Tolu O. Occurrence of ESBL and MBL in clinical isolates of Pseudomonas aeruginosa from Lagos, Nigeria. J Am Sci 2007;3(4):81-5.

[36] Chaudhary U, Bhaskar H, Sharma M. Imipenem-EDTA disk method for rapid identification of metallo-betalactamase producing Gram-negative bacteria. Indian Journal of Medical Research 2008;127(4):406-7.

[37] Anil C, Shahid RM. Antimicrobial susceptibility patterns of Pseudomonas aeruginosa clinical isolates at a tertiary care hospital in Kathmandu, Nepal. Asian J Pharm Clin Res 2013;6(3):235-8.

[38] Juan C, Zamorano L, Mena A, et al. Metallo- $\beta$-lactamaseproducing Pseudomonas putida as a reservoir of multidrug resistance elements that can be transferred to successful Pseudomonas aeruginosa clones. Journal of Antimicrobial Chemotherapy 2010;65(3):474-8. 